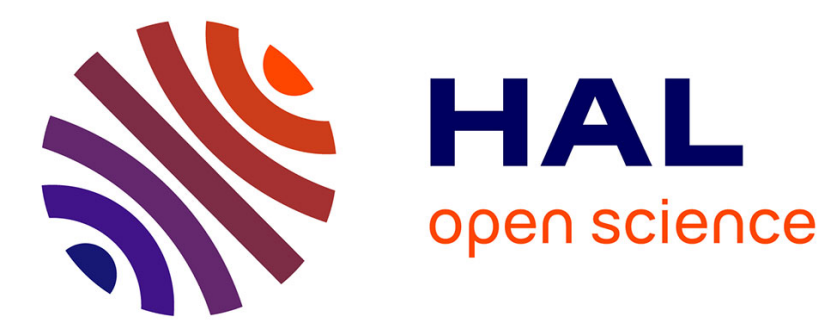

\title{
Étude des variations de fréquence fondamentale relatives au genre chez des bilingues Anglais/Français
}

\author{
Erwan Pépiot, Aron Arnold
}

\section{To cite this version:}

Erwan Pépiot, Aron Arnold. Étude des variations de fréquence fondamentale relatives au genre chez des bilingues Anglais/Français. XXIIe Journées d'Études sur la Parole, Jun 2018, Aix-En-Provence, France. pp. 178-186, 10.21437/jep.2018-21 . hal-03004728

\section{HAL Id: hal-03004728 \\ https://hal.science/hal-03004728}

Submitted on 13 Nov 2020

HAL is a multi-disciplinary open access archive for the deposit and dissemination of scientific research documents, whether they are published or not. The documents may come from teaching and research institutions in France or abroad, or from public or private research centers.
L'archive ouverte pluridisciplinaire HAL, est destinée au dépôt et à la diffusion de documents scientifiques de niveau recherche, publiés ou non, émanant des établissements d'enseignement et de recherche français ou étrangers, des laboratoires publics ou privés. 


\title{
Étude des variations de fréquence fondamentale relatives au genre chez des bilingues Anglais/Français
}

\author{
Erwan Pépiot ${ }^{1}$, Aron Arnold ${ }^{2}$ \\ (1) TransCrit (groupe LECSeL), 2 rue de la liberté, 93526 Saint-Denis, France \\ (2) VALIBEL - Université Catholique de Louvain, Place Blaise Pascal 1, 1348 Louvain-la- \\ Neuve, Belgique \\ erwan.pepiotefree.fr, aron.arnoldeuclouvain.be
}

\section{RESUME}

La présente étude porte sur les productions de locutrices et locuteurs bilingues anglais/français lors d'une tâche de lecture de phrases et en parole semi-spontanée. La fréquence fondamentale (F0) moyenne, la plage de variation de F0 et l'écart-type de F0 ont été mesurés dans les deux langues. Les résultats indiquent un effet significatif des facteurs langue et genre sur l'ensemble de ces paramètres. La F0 moyenne est globalement plus élevée en français; la modulation de F0 est quant à elle globalement plus forte en anglais. Indépendamment de la langue, les locutrices présentent une F0 moyenne plus élevée. En outre, les locutrices ont plus fortement augmenté leur F0 moyenne lors de l'emploi du français et les locuteurs ont réduit plus fortement leur modulation dans cette langue. Ces données suggèrent l'existence de normes vocales relatives au genre différentes dans les deux langues étudiées.

\begin{abstract}
A study of fundamental frequency in female and male English/French bilingual speakers.

The present study deals with the productions of English/French bilingual speakers in a reading task and in semi-spontaneous speech. Mean fundamental frequency, F0 range and F0 standard-deviation were measured in both languages. Results show a significant effect of gender and language on all these parameters. Overall, mean F0 was higher in French while F0 modulation was stronger in English. Regardless of language, female speakers exhibited higher F0 than males. Moreover, the increase of mean F0 in French was larger in female speakers. On the other hand, the decrease of F0 modulation in French was stronger for male speakers. These data support the idea of language- and gender-specific vocal codes, to which bilingual speakers seem to adapt.
\end{abstract}

MOTS-CLES : fréquence fondamentale, intonation, bilinguisme, voix et genre, variations interlangues.

KEYWORDS: fundamental frequency, intonation, bilingualism, voice and gender, cross-language variation.

\section{Introduction}

Les différences vocales entre femmes et hommes ont fait l'objet de multiples études phonétiques au cours des dernières décennies. Ces études se focalisent en grande partie sur la fréquence fondamentale $(\mathrm{F} 0)$, considérée avec les fréquences de résonance comme un paramètre décisif dans ce 
qui constitue une voix en voix de femme ou en voix d'homme. S'il existe diverses études qui comparent les F0 moyennes de locuteur·ice·s de différentes langues (p. ex. Traunmüller, Eriksson 1995), peu d'études ont pris comme objet les variations intra-individuelles de bilingues lors du passage d'une langue à l'autre et analysé les éventuelles différences genrées dans ces variations. Nous suggérons que l'étude de ces variations permet non seulement de s'éloigner d'une vision statique de la F0, dans laquelle celle-ci est présentée comme une caractéristique essentielle des locuteur·ice·s ou comme principalement due à leur anatomie, pour en adopter une vision dynamique qui permet d'intégrer des questions de différences culturelles de normes de genre et de performances du genre.

Au niveau acoustique, la F0 des voix d'hommes se situe généralement dans des fréquences plus basses que celles des voix de femmes (Boë et al., 1975). Ces différences acoustiques sont en partie dues aux différences sexuées qui émergent lors de la puberté dans les appareils phonatoires. Les taux de testostérone, de progestérone et d'estrogènes varient en fonction de la sexuation du corps et favorisent un développement de plis vocaux plus massifs dans les corps de sexe masculin que dans ceux de sexe féminin (Kahane, 1978 ; Abitbol et al., 1999), ce qui explique en partie pourquoi les plis vocaux des hommes vibrent généralement à une fréquence plus basse que ceux des femmes. À côté des hormones, d'autres facteurs tels que l'âge (Honjo, Isshiki, 1980) ou la consommation de cigarettes (Matar, 2016) peuvent modifier la masse des plis vocaux et ainsi provoquer un abaissement ou une élévation de la F0.

Dans la voix, les facteurs anatomiques et sociaux sont cependant inextricables. Il est désormais établi que la voix participe à la construction sociale des identités de genre (Arnold, 2015 ; Pépiot, 2014b). Chaque locuteur ·ice a un appareil phonatoire d'une forme donnée (qui joue sur la F0 et les fréquences de résonance produites), mais fait un usage de cet appareil en fonction de son genre. Une voix n'est ainsi jamais uniquement le reflet d'une anatomie, mais aussi le résultat d'une performance genrée: les femmes mobilisent certaines pratiques articulatoires afin de produire des voix relativement aigues et claires, et les hommes en utilisent d'autres afin de produire des voix relativement graves et sombres (Arnold, 2016).

Dans la littérature, la plage de variation et les modulations de F0 sont elles aussi souvent décrites comme indexant des catégories de genre: les femmes utiliseraient des plages de variations plus étendues que les hommes et moduleraient plus (p. ex. Austin, 1965 ; Lakoff, 1975, p. 56). Ceci a cependant été contredit par certaines études portant sur l'anglais étatsunien. Par exemple, Henton $(1989$; 1995) a montré que si on utilise une échelle de mesure en demi-tons, c'est-à-dire une mesure logarithmique qui reflète la perception humaine des variations de hauteur, alors les différences entre femmes et hommes s'effacent. Pépiot (2014a), en utilisant le même procédé de mesure que Henton a cependant trouvé que les locutrices françaises modulaient plus que les locuteurs français. Ces différences de résultats suggèrent qu'il existe des pratiques genrées différentes concernant la modulation chez les locutrices et locuteurs étatsunien et français.

Qu'en est-il alors des locuteur·ice's multilingues? Comment s'adaptent-elles/ils aux normes genrées des différentes langues parlées? En quoi ces normes diffèrent-elles pour les femmes et les hommes ? Ces questions n'ont pour l'instant fait l'objet que de peu d'attention.

Différentes études réalisées sur des locuteur·ice·s bilingues ont montré qu'en fonction de la langue parlée, ces dernier·e·s vont varier leur F0 moyenne (Altenberg, Ferrand, 2006 ; Lee, Van Lanker Sidtis, 2017) ainsi que leur plage de variation de F0 (Mennen et al., 2012). Par exemple, l'étude d'Altenberg et Ferrand (2006) montre que des locutrices bilingues russes L1 / anglais L2 tendent à parler avec une F0 plus basse en anglais. Cette analyse a cependant été conduite uniquement sur des productions de locutrices - il est conséquemment impossible de savoir si les variations observées relèvent d'une adaptation aux normes genrées des différentes langues, ou tout simplement de pratiques liées aux langues elles-mêmes, sans considération de genre. 
Nous avons donc souhaité, par la présente étude, investiguer les pratiques de locutrices et de locuteurs bilingues anglais L1 / français L2, en mesurant leur F0 dans différentes conditions (lecture de phrases et parole spontanée). Notre hypothèse de départ est la suivante : les locutrices et les locuteurs bilingues adaptent leurs pratiques vocales aux normes genrées de la langue employée.

\section{Méthode}

\subsection{Corpus}

La présente étude se base sur l'analyse d'un corpus en anglais et en français collecté lors de deux tâches distinctes. Ces deux tâches ont permis de collecter des séquences de parole lue et de parole semi-spontanée.

La première tâche consistait en une lecture de 12 phrases en anglais (telles que «When the weather is cold and rainy, I'd rather stay at home. "; "My sister told me she'd come by tomorrow. " ou encore "If you do that again, I'll call the police! ») et 12 phrases similaires en français ("Quand il fait froid et qu'il pleut, je préfère rester chez moi. » ; " Ma soeur m'a dit qu'elle allait passer demain. "; "Si tu refais ça, j'appelle la police! »; etc.).

La deuxième tâche, qui a permis de collecter de la parole semi-spontanée, consistait en une narration d'un évènement passé - les dernières vacances. La narration en anglais a été amorcée par l'énoncé "Tell me about your last vacation », et celle en français par l'énoncé "Parlez-moi de vos dernières vacances ».

\subsection{Participant $\cdot e \cdot s$}

Six locutrices et six locuteurs bilingues anglais L1 / français L2 ont pris part à cette étude. Les participant $\cdot e \cdot s$ sont originaires du nord-est des États-Unis et vivent en région parisienne depuis plusieurs années. Tou·te's font état d'une pratique quotidienne de la langue française et d'un niveau d'aisance dans cette langue supérieur ou égal à 3, sur une échelle allant de 0 à 5 , via un questionnaire inspiré par celui de Grosjean (2013). Nous reprenons également à notre compte la définition du bilinguisme proposé par cet auteur.

Les participant $\cdot \mathrm{e} \cdot \mathrm{s}$ étaient âgé $\cdot \mathrm{e} \cdot \mathrm{s}$ de 29 à 54 ans $(S D=7,6$ ans) au moment des enregistrements, avec une moyenne d'âge de 41,8 ans pour les femmes et de 40 ans pour les hommes. Aucun·e n'était fumeur·euse et ne présentait de troubles de la parole. Une clé USB a été offerte en échange de la participation à la présente étude.

\subsection{Procédure d'enregistrement}

Les enregistrements se sont déroulés dans une chambre anéchoïque avec un enregistreur numérique Edirol R09-HR de marque Roland. Chaque session d'enregistrement comprenait les tâches détaillées dans la section 2.1 : en premier lieu, la lecture des phrases avec un débit de parole moyen (deux lectures pour chaque item), puis la narration portant sur les dernières vacances. Afin de neutraliser de possibles biais liés à l'ordre d'emploi des deux langues (voir Altenberg, Ferrand, 2006), la moitié des locuteur·ice's a commencé par effectuer ces tâches en langue française, et l'autre moitié en langue anglaise. 


\subsection{Analyse des données}

L'analyse acoustique du corpus a été effectuée à l'aide du logiciel Praat (Boersma, 2017). Les paramètres suivants ont été relevés pour chacune des phrases ainsi que pour la parole spontanée :

- $\quad$ F0 moyenne.

- Plage de variation de F0, qui correspond à l'écart entre la fréquence la plus basse et la fréquence la plus haute atteinte à l'intérieur d'une unité linguistique donnée.

- Écart-type de F0, qui constitue le paramètre le plus en mesure de rendre compte de la modulation de F0, en particulier lors de l'étude de longues séquences de parole continue.

Ces données ont été obtenues en créant pour chaque phrase/discours un fichier Pitch sur Praat, puis en collectant les valeurs dans la fenêtre Pitch info. La plage de variation de F0 ainsi que l'écarttype ont été mesurés en Hertz mais également en demi-tons. Cette échelle est en effet particulièrement pertinente car elle rend compte de la variation de hauteur perçue (Henton, 1995).

Les données ainsi recueillies ont ensuite fait l'objet de tests statistiques de type ANOVA, dans le but de tester l'influence des facteurs « langue parlée » et « genre des locuteur·ice·s».

\section{Résultats}

\subsection{Phrases lues}

La F0 moyenne des locutrices et des locuteurs sur les phrases lues, en anglais et en français, est présentée dans le tableau 1 ci-après.

F0 moyenne - Phrases lues (Hz)

\begin{tabular}{crrr} 
Loc. & Anglais & \multicolumn{1}{c}{ Français } & \multicolumn{1}{c}{ \% diff. FR/AN } \\
\hline F1 & 195 & 211 & $\mathbf{+ 8 , 2 8}$ \\
\hline F2 & 224 & 234 & $\mathbf{+ 4 , 2 9}$ \\
\hline F3 & 176 & 192 & $\mathbf{+ 8 , 6 8}$ \\
\hline F4 & 201 & 218 & $\mathbf{+ 8 , 3 7}$ \\
\hline F5 & 186 & 205 & $\mathbf{+ 1 0 , 2 0}$ \\
\hline F6 & 187 & 206 & $\mathbf{+ 1 0 , 0 1}$ \\
\hline Moyenne F & $\mathbf{1 9 5}$ & $\mathbf{2 1 1}$ & $\mathbf{+ 8 , 1 7}$ \\
\hline H1 & 113 & 112 & $\mathbf{- 1 , 2 9}$ \\
\hline H2 & 81 & 83 & $\mathbf{+ 2 , 6 3}$ \\
\hline H3 & 120 & 121 & $\mathbf{+ 1 , 1 1}$ \\
\hline H4 & 106 & 103 & $\mathbf{- 3 , 4 9}$ \\
\hline H5 & 129 & 129 & $\mathbf{- 0 , 1 0}$ \\
\hline H6 & 108 & 119 & $\mathbf{+ 9 , 7 7}$ \\
\hline Moyenne H & $\mathbf{1 1 0}$ & $\mathbf{1 1 1}$ & $\mathbf{+ 1 , 3 3}$ \\
\hline
\end{tabular}

TABLEAU 1 : F0 moyenne en Hertz (Hz) des locutrices et des locuteurs sur les phrases lues $(12$ x 2 occurrences), en fonction de la langue parlée (anglais ou français).

On constate que les locutrices présentent toutes une F0 moyenne plus élevée en français qu'en anglais. Cette élévation du F0 est de $8,17 \%$ en moyenne, toutes locutrices confondues. Chez les 
locuteurs, en revanche, il est difficile de dégager une tendance claire : trois d'entre eux présentent une F0 plus élevée en français, mais l'inverse est vrai pour les trois autres.

Une ANOVA à deux facteurs ( « langue parlée » et « genre ») confirme l'influence significative de la langue, avec $\mathrm{F}(1,572)=25,566$ et $\mathrm{p}<0,0001$, et du genre des locuteur $\cdot$ ice $\cdot \mathrm{s}$, avec $\mathrm{F}(1,572)=2897,3$ et $\mathrm{p}<0,0001$, sur la F0 moyenne. De plus, on observe une interaction significative entre les deux facteurs $(\mathrm{F}(1,572)=17,712 ; \mathrm{p}<0,0001)$. Cela indique que les locutrices et les locuteurs n'ont pas adapté de la même manière leur F0 moyenne en passant d'une langue à l'autre, suggérant ainsi l'existence d'une variation inter-genre sur l'utilisation de ce paramètre acoustique en fonction de la langue parlée.

La plage de variation de F0, en Hertz et demi-tons, ainsi que son écart-type moyen (SD) en Hertz $(\mathrm{Hz})$ et demi-tons (dt) sur les phrases lues sont visibles ci-dessous, dans le tableau 2.

\begin{tabular}{|c|c|c|c|c|c|c|c|c|c|}
\hline \multirow[b]{2}{*}{ Loc. } & \multicolumn{4}{|c|}{ Phrases lues - AN } & \multicolumn{4}{|c|}{ Phrases lues - FR } & \multirow[b]{2}{*}{$\begin{array}{c}\% \text { diff. FR/AN } \\
\text { SD (dt) }\end{array}$} \\
\hline & $\begin{array}{l}\text { Pl. var. } \\
\text { (Hz) }\end{array}$ & $\begin{array}{l}\text { Pl. var } \\
\text { (dt) }\end{array}$ & $\begin{array}{c}\text { SD } \\
(\mathbf{H z})\end{array}$ & SD & $\begin{array}{l}\text { Pl. var. } \\
\text { (Hz) }\end{array}$ & $\begin{array}{l}\text { Pl. var. } \\
\text { (dt) }\end{array}$ & $\begin{array}{l}\text { SD } \\
(\mathrm{Hz})\end{array}$ & $\begin{array}{l}\text { SD } \\
\text { (dt) }\end{array}$ & \\
\hline F1 & 218,28 & 20,37 & 50,56 & 4,85 & 219,36 & 19,06 & 40,53 & 3,60 & $-25,76$ \\
\hline F2 & 233,25 & 20,21 & 46,37 & 3,79 & 203,33 & 15,92 & 39,46 & 2,97 & $-21,76$ \\
\hline F3 & 166,34 & 16,71 & 32,56 & 3,22 & 165,56 & 14,95 & 25,24 & 2,29 & $-28,90$ \\
\hline F4 & 201,91 & 17,93 & 38,39 & 3,23 & 224,59 & 19,56 & 41,46 & 3,54 & $+9,60$ \\
\hline F5 & 182,75 & 16,61 & 38,00 & 3,45 & 162,96 & 14,00 & 33,85 & 2,88 & $-16,54$ \\
\hline F6 & 173,64 & 16,88 & 28,12 & 2,68 & 213,91 & 18,91 & 33,74 & 2,92 & $+8,88$ \\
\hline Moy. F & 196,03 & 18,12 & 39,00 & 3,54 & 198,28 & 17,07 & 35,71 & 3,03 & $-14,26$ \\
\hline H1 & 101,92 & 15,30 & 23,61 & 3,51 & 88,56 & 13,96 & 19,00 & 3,00 & $-14,59$ \\
\hline $\mathbf{H 2}$ & 54,12 & 10,68 & 9,78 & 2,01 & 53,50 & 10,47 & 10,53 & 2,14 & $+6,27$ \\
\hline H3 & 91,55 & 13,82 & 22,03 & 3,24 & 82,75 & 11,49 & 19,19 & 2,62 & $-19,05$ \\
\hline H4 & 79,84 & 12,50 & 20,13 & 3,16 & 72,43 & 11,86 & 16,91 & 2,79 & $-11,55$ \\
\hline H5 & 94,08 & 11,95 & 24,41 & 3,13 & 78,71 & 10,32 & 18,48 & 2,41 & $-23,25$ \\
\hline H6 & 76,66 & 12,98 & 16,85 & 2,73 & 69,23 & 9,94 & 14,87 & 2,06 & $-24,84$ \\
\hline Moy. H & 83,03 & 12,87 & 19,47 & 2,96 & 74,20 & 11,34 & 16,50 & 2,50 & $-15,61$ \\
\hline
\end{tabular}

TABLEAU 2 : Valeurs moyennes de la plage de variation de F0 en Hertz (Hz) et demi-tons (dt) et de l'écart-type (SD) de F0 en Hertz et demi-tons obtenus par les locutrices (F) et les locuteurs (H) sur les phrases lues (12 x 2 occurrences), en fonction de la langue parlée (anglais ou français).

Les données recueillies montrent que plage de variation est réduite lors des séquences en français. Cette réduction est de $11,89 \%$ (en dt) chez les locuteurs et de $14,36 \%$ (en dt) chez les locutrices.

On note également une tendance globale à une plus faible modulation lors de l'emploi du français. Ce phénomène est plus prononcé chez les locuteurs, avec une diminution de 15,61\% de l'écart-type (en dt), contre 14,26\% chez les locutrices.

Une ANOVA à deux facteurs ( «langue parlée » et "genre ») a été conduite sur la plage de variation de F0 (en dt). L'analyse confirme un rôle significatif de la langue, avec $F(1,572)=18,823$ et $p<0,0001$, et du genre des locuteur.rices, avec $F(1,572)=340,109$ et $p<0,0001$. Il en va de même sur l'écart-type, exprimé en demi-tons, pour le facteur langue $(\mathrm{F}(1,572)=44,087 ; \mathrm{p}<0,0001)$ et pour le facteur genre $(\mathrm{F}(1,572)=57,530 ; \mathrm{p}<0,0001)$. 


\subsection{Parole semi-spontanée}

Comme expliqué supra, les locutrices et locuteurs ont outre la tâche de lecture de phrases également eu à produire des séquences de parole semi-spontanée. Les F0 moyennes des participant $\cdot e \cdot s$ lors de ces séquences, qui avaient des durées d'une à deux minutes, sont présentées dans le tableau 3.

\begin{tabular}{crrr} 
& \multicolumn{3}{c}{ F0 moyenne - Discours semi-spontané (Hz) } \\
\cline { 2 - 4 } Loc. & \multicolumn{1}{c}{ Anglais } & Français & \% diff. FR/AN \\
\hline F1 & 179 & 189 & $\mathbf{+ 5 , 4 7}$ \\
\hline F2 & 190 & 195 & $\mathbf{+ 2 , 9 5}$ \\
\hline F3 & 167 & 175 & $+\mathbf{4 , 7 3}$ \\
\hline F4 & 193 & 197 & $+\mathbf{2 , 1 3}$ \\
\hline F5 & 173 & 177 & $+\mathbf{2 , 2 5}$ \\
\hline F6 & 184 & 182 & $\mathbf{- 0 , 9 8}$ \\
\hline Moy. F & $\mathbf{1 8 1}$ & $\mathbf{1 8 6}$ & $\mathbf{+ 2 , 7 6}$ \\
\hline H1 & 104 & 105 & $\mathbf{+ 0 , 8 6}$ \\
\hline H2 & 74 & 73 & $\mathbf{- 1 , 6 2}$ \\
\hline H3 & 103 & 105 & $\mathbf{+ 2 , 4 3}$ \\
\hline H4 & 99 & 99 & $\mathbf{+ 0 , 2 0}$ \\
\hline H5 & 121 & 121 & $\mathbf{+ 0 , 5 0}$ \\
\hline H6 & 99 & 100 & $\mathbf{+ 1 , 5 2}$ \\
\hline Moy. H & $\mathbf{1 0 0}$ & $\mathbf{1 0 1}$ & $\mathbf{+ 0 , 6 5}$ \\
\hline
\end{tabular}

TABLEAU 3 : F0 moyenne des locutrices et des locuteurs sur le discours semi-spontané, en fonction de la langue parlée (anglais ou français).

Les résultats obtenus vont dans le sens des tendances observées sur les phrases lues. En effet, cinq des six locutrices ont utilisé une F0 plus élevée lorsqu'elles parlaient français. La sixième présente quant à elle des F0 relativement stables dans les deux langues ( $-0,98 \%$ en français). Toutes locutrices confondues, on observe une augmentation de la F0 moyenne de 2,76\% en français, comparée à l'anglais. Chez les locuteurs, on constate une relative stabilité de la F0 moyenne avec des variations de $+0,65 \%$.

Une ANOVA à deux facteurs (« langue parlée » et « genre ») confirme l'influence significative de la langue $(F(1,476)=7.059 ; p<0,01)$ et du genre des locuteur $\cdot$ ice $\cdot s(F(1,476)=6062,193 ; p<0,0001)$ sur la F0 moyenne. A l'instar des phrases lues, on observe ici aussi une interaction entre les deux facteurs $(\mathrm{F}(1,476)=3,816 ; \mathrm{p}=0,0513)$, même si celle-ci n'atteint pas le seuil de significativité.

Le tableau 4, ci-après, présente la plage de variation de F0 (en $\mathrm{Hz}$ et dt), ainsi que l'écart-type (également en $\mathrm{Hz}$ et dt) lors des séquences en parole semi-spontanée. 


\begin{tabular}{|c|c|c|c|c|c|c|c|c|c|}
\hline \multirow[b]{2}{*}{ Loc. } & \multicolumn{4}{|c|}{ Discours semi-spontané - AN } & \multicolumn{4}{|c|}{ Discours semi-spontané - FR } & \multirow[b]{2}{*}{$\begin{array}{c}\% \text { diff. FR/AN } \\
\text { SD (dt) }\end{array}$} \\
\hline & $\begin{array}{l}\text { Pl. var. } \\
(\mathrm{Hz})\end{array}$ & $\begin{array}{l}\text { Pl. var. } \\
\text { (dt) }\end{array}$ & $\begin{array}{l}\mathrm{SD} \\
(\mathrm{Hz})\end{array}$ & $\begin{array}{l}\text { SD } \\
\text { (st) }\end{array}$ & $\begin{array}{l}\text { Pl. var. } \\
(\mathrm{Hz})\end{array}$ & $\begin{array}{l}\text { Pl. var. } \\
\text { (dt) }\end{array}$ & $\begin{array}{l}\mathrm{SD} \\
(\mathrm{Hz})\end{array}$ & $\begin{array}{l}\text { SD } \\
(d t)\end{array}$ & \\
\hline F1 & 300,32 & 25,41 & 42,62 & 3,94 & 297,94 & 25,30 & 41,65 & 3,54 & $-10,15$ \\
\hline F2 & 309,61 & 25,82 & 34,26 & 3,24 & 305,69 & 25,64 & 36,92 & 3,34 & $+3,09$ \\
\hline F3 & 211,16 & 20,89 & 22,51 & 2,23 & 253,20 & 23,05 & 23,44 & 2,18 & $-2,24$ \\
\hline F4 & 289,14 & 23,41 & 28,16 & 2,52 & 276,55 & 23,57 & 28,38 & 2,46 & $-2,38$ \\
\hline F5 & 300,88 & 25,42 & 40,94 & 3,64 & 306,62 & 25,61 & 42,90 & 3,52 & $-3,30$ \\
\hline F6 & 244,63 & 22,63 & 30,98 & 2,75 & 286,52 & 24,78 & 29,70 & 2,60 & $-5,45$ \\
\hline Moy. F & 275,96 & 23,93 & 33,25 & 3,05 & 287,75 & 24,66 & $\mathbf{3 3 , 8 3}$ & 2,94 & $-3,41$ \\
\hline H1 & 224,44 & 28,21 & 34,33 & 4,21 & 177,13 & 28,42 & 26,78 & 3,56 & $-15,44$ \\
\hline H2 & 67,52 & 16,27 & 7,13 & 1,58 & 53,24 & 12,52 & 6,87 & 1,54 & $-2,53$ \\
\hline H3 & 124,70 & 24,24 & 21,91 & 4,36 & 151,39 & 21,68 & 13,37 & 2,09 & $-52,06$ \\
\hline H4 & 155,71 & 24,44 & 20,11 & 3,18 & 151,03 & 24,08 & 7,53 & 2,95 & $-7,23$ \\
\hline H5 & 179,88 & 21,87 & 25,44 & 3,21 & 176,18 & 21,78 & 20,98 & 2,67 & $-16,82$ \\
\hline H6 & 127,53 & 19,71 & 11,87 & 1,94 & 107,71 & 17,79 & 11,75 & 1,92 & $-1,03$ \\
\hline Moy. H & 146,63 & 22,46 & 20,13 & 3,08 & 136,11 & 21,05 & 14,55 & 2,46 & $-15,85$ \\
\hline
\end{tabular}

TABLEAU 4 : Valeurs moyennes de la plage de variation de F0 (en $\mathrm{Hz}$ et dt) et de l'écart-type de F0 (en $\mathrm{Hz}$ et dt) obtenus par les locutrices (F) et les locuteurs (M) en parole semi-spontanée, en fonction de la langue parlée (anglais ou français).

Si l'on s'intéresse aux différences de l'écart-type de la F0 exprimé en demi-tons entre les deux langues (dernière colonne du tableau 4 ), on constate que tou $\cdot$ te $\cdot s$ les participant $\cdot e \cdot s$, à l'exception d'une locutrice, ont moins modulé leur fréquence fondamentale en français qu'en anglais. Cependant, on note que cette tendance est nettement plus marquée chez les locuteurs, avec un écart-type de $15,85 \%$ inférieur en français, alors que chez les locutrices, la diminution n'est que de 3,41\%. Ce résultat rejoint ainsi celui observé sur les phrases lues.

Une ANOVA à deux facteurs (« langue parlée » et «genre ») a été effectuée sur le paramètre de l'écart-type. Elle met en évidence un rôle significatif de la langue, avec $\mathrm{F}(1,476)=29,353$ et $\mathrm{p}<0,0001$, et du genre des locuteur·ice $\cdot \mathrm{s}$, avec $\mathrm{F}(1,476)=11,371$ et $\mathrm{p}<0,001$. De plus, on observe une interaction significative entre les deux facteurs $(F(1,476)=14,097 ; p<0,001)$, ce qui indique que les locutrices et les locuteurs n'ont pas adapté de la même manière la modulation de leur fréquence fondamentale en passant d'une langue à l'autre. Ainsi, la modulation de F0 était globalement similaire chez les locutrices et les locuteurs lors de l'emploi de la langue anglaise - 3,05 demi-tons chez les locutrices et 3,08 demi-tons chez les locuteurs. En revanche, lors des séquences en langue française, les femmes ont plus modulé leur F0 que les hommes - l'écart-type des locutrices est de 2,94 demitons et celui des locuteurs de 2,46.

\section{Conclusion / discussion}

Comme il a été indiqué dans la section précédente, nous avons trouvé une interaction significative entre les facteurs langue et genre sur la F0 moyenne en parole lue et sur l'écart-type de F0 en parole spontanée. On observe également une tendance similaire sur la F0 moyenne en parole spontanée, même si l'interaction entre les deux facteurs étudiés n'atteint pas le seuil de significativité. Ceci indique que dans ces contextes, la langue va globalement jouer sur ces paramètres acoustiques, mais de manière différente en fonction du genre des locuteur $\cdot$ ice $\cdot s$. 
L'analyse de la F0 moyenne a montré que celle-ci est globalement plus élevée en français qu'en anglais, indépendamment du genre des locuteur·ice·s. Cependant, si l'on compare les locutrices et les locuteurs, on constate une différence : lors de l'emploi du français, on trouve une augmentation de la F0 moyenne en parole lue chez toutes les locutrices et en parole spontanée chez 5 sur 6 locutrices, alors qu'on retrouve cette augmentation moins régulièrement chez les locuteurs - ces derniers ont utilisé une F0 moyenne similaire dans les deux langues en parole spontanée et seulement 3 des 6 locuteurs l'ont augmentée en français en parole lue. Le fait que les locutrices et les locuteurs n'aient pas adapté de la même manière leur F0 moyenne en passant d'une langue à l'autre peut être interprété comme un indice d'une différence de genre ethnolinguistique dans l'utilisation de ce paramètre acoustique.

Pour ce qui est des modulations, l'écart-type de la F0 exprimé en demi-tons montre que tou·te·s les participant·e·s, à l'exception d'une seule locutrice, ont moins modulé leur F0 en français qu'en anglais. Cette réduction de modulation est plus prononcée chez les locuteurs, avec une diminution de $15,61 \%$ en parole lue et de $15,85 \%$ en parole spontanée, contre respectivement $14,36 \%$ chez les locutrices en parole lue et 3,41\% en parole spontanée. En outre, nous avons remarqué que lors des séquences produites en anglais en parole spontanée, les locuteurs ont autant modulé leur F0 que les locutrices. Ce résultat va dans le sens de l'étude de Henton (1995) qui, en mesurant les modulations de locuteurs féminins et masculins étasuniens en demi-tons, n'a trouvé aucune différence inter-genres significative. En revanche, lors des séquences en français en parole spontanée, on note que les locuteurs modulaient nettement moins leur F0 que les locutrices. Ces résultats confirment ceux de Pépiot (2014a) et suggèrent qu'en français, les modulations de F0 font partie des pratiques vocales qui contribuent à produire de la différence entre le groupe des femmes et celui des hommes et à diminuer les différences à l'intérieur de ces groupes.

L'analyse de ces différents paramètres, ainsi que l'étude croisée des facteurs langue et genre sur les productions de bilingues apporte ainsi de nouveaux éléments qui n'avaient pas pu être dégagés des études précédentes, comme celles de Altenberg et Ferrand (2006), Lee et Van Lanker Sidtis (2017) ou Mennen et al. (2012). La présente étude révèle notamment que la production d'une voix de femme ou d'homme mobilise des pratiques vocales différentes en fonction de la langue et confirme ainsi que la F0 n'est pas une caractéristique essentielle des locuteur·ice's, principalement due à l'anatomie de leur appareil phonatoire, mais qu'elle résulte également d'un apprentissage et d'une socialisation en tant que membre d'une catégorie de genre spécifique. Ceci constitue un argument pour s'éloigner d'une conception anatomiste de la F0 que l'on peut retrouver fréquemment dans la littérature phonétique, et pour une plus grande considération des facteurs sociaux dans l'étude de la voix et de la parole.

Parmi les limites de cette étude, on citera notamment le nombre relativement réduit de participant $\cdot \mathrm{e} \cdot \mathrm{s}$. Afin de confirmer nos résultats, la présente étude pourrait être répliquée avec un nombre plus important de locuteur·ice·s. D'autre part, nous avons ici uniquement analysé de la parole produite par des bilingues anglais L1 / français L2. Il serait opportun d'étudier également la parole de bilingues français L1 / anglais L2. En effet, l'augmentation de la F0 moyenne en français pourrait éventuellement être liée, dans une certaine mesure, à un stress éprouvé par les locuteur·ice·s lors de la prise de parole dans leur langue seconde - le stress induisant régulièrement une augmentation de F0 (Scherer 1986). La comparaison avec les productions de bilingues français L1 / anglais L2 permettrait d'établir si la prise de parole en langue seconde agit sur la F0.

\section{Remerciements}

Un grand merci à tou·te·s les locutrices et locuteurs ayant pris part à cette étude, ainsi qu'à Juliette Stockman qui a participé à la préparation et à l'analyse de notre corpus. 


\section{Références}

Aвiтbol J., Aвitbol P., Aвiтвоl B. (1999). Sex hormones and the female voice. Journal of Voice $13,424-446$.

Altenberg E. P., FerRAND C. T. (2006). Fundamental frequency in monolingual English, bilingual English/Russian, and bilingual English/Cantonese young adult women. Journal of Voice 20(1), 8996.

ARNOLD A. (2015). Voix et transidentité : changer de voix pour changer de genre ?. Langage et société 151(1), 87-105.

ARnold A. (2016). Voix. Encyclopédie critique du genre, 713-721. Paris : La Découverte.

Austin W. M. (1965). Some social aspects of paralanguage. Canadian Journal of Linguistics/Revue canadienne de linguistique 11(1), 31-39.

Boersma P., WeENINK D. (2017). Praat: doing phonetics by computer [Logiciel]. Version 6.0.36, publiée le 11 Novembre 2017 sur le site www.praat.org

BoË L.-J., CONTINI M., RAKOTOFIRINGA H. (1975). Etude statistique de la fréquence laryngienne. Phonetica 32(1), 1- 23.

Grosjean, F. \& LI, P. (2013). The Psycholinguistics of Bilingualism. Oxford : Wiley-Blackwell.

HENTON C. (1989). Fact and fiction in the description of female and male pitch. Language \& Communication, 9(4), 299-311.

Henton C. (1995). Pitch dynamism in female and male speech. Language \& Communication 15(1), 43-61.

Honjo I., ISSHIKI N. (1980). Laryngoscopic and Voice Characteristics of Aged Persons. Archives of Otolaryngology 106(3), 14-150.

KAHANE J. C. 1978. A morphological study of the human prepubertal and pubertal larynx. American Journal of Anatomy 151, 11-19.

LAKOFF R. (1975). Language and Woman's Place. New York : Harper \& Row.

LEE B., VAN LANCKER SIDTIS D. (2017). The bilingual voice: Vocal characteristics when speaking two languages across speech tasks. Speech, Language and Hearing 20(3), 174-185.

Matar N., Portes C., Lancia L., Legou T., Baider F. (2016). Voice Quality and Gender Stereotypes: A Study of Lebanese Women With Reinke's Edema. Journal of Speech, Language, and Hearing Research 59(6), S1608-S1617.

MENNEN I., SchaefFler F., DOCHERTY G. (2012). Cross-language differences in fundamental frequency range: A comparison of English and German. The Journal of the Acoustical Society of America 131(3), 2249-2260.

PEPIOT E. (2014a). Male and female speech: a study of mean F0, F0 range, phonation type and speech rate in Parisian French and American English speakers. Proceedings of the 7th International Conference on Speech Prosody, 305-309.

PEPIOT, E. (2014b). Voix et genre : un état de la question. In Ibrahim, A.H. (éd.), La langue, la voix, la parole (pp. 53-86), Paris : CRL.

SCHERER K. R. (1986). Voice, Stress, and Emotion. Dynamics of Stress. Boston : Springer

TRAUNMÜLLER H., ERIKSSON A. (1995). The frequency range of the voice fundamental in the speech of male and female adults. Manuscrit : http://www2.ling.su.se/staff/hartmut/f0_m\&f.pdf. 